Gut, 1971, 12, 350-355

\title{
Movement of segmental constrictions in the human colon
}

\author{
JAMES A. RITCHIE ${ }^{1}$ \\ From the Nuffield Department of Clinical Medicine and the Nuffield Institute for Medical Research, Oxford
}

SUMMARY Changes in the segmental pattern of opaque contents in the transverse colon among patients with diverticulosis were observed by means of time-lapse cinefluorography after ingestion of barium sulphate suspension.

In some of them the muscular constrictions separating the opaque masses of bowel contents were seen to relax and later to re-form in a different spatial relationship to fixed points on the bowel wall represented by the necks of diverticula. In other instances ring contractions moved progressively along the bowel wall, temporarily occluding the necks of any diverticula they passed. Bowel contents were seen to be transported ahead of the advancing constrictions at varying rates and in either direction.

When human subjects are examined radiographically after ingestion of barium sulphate, their colonic contents are usually seen to be subdivided by annular constrictions into a series of opaque segmental masses. Although, under these conditions of observation, the bowel wall itself is not visible, the constrictions may be assumed to have been caused by localized contractions of its circular muscle layer.

These segmental constrictions, sometimes referred to as interhaustral folds, may be obliterated under certain circumstances as, for example, in the preliminary stages of a mass propulsive movement. Whether, after relaxing in this way, the constrictions necessarily re-form at the same points in the bowel or whether they can readily move to other situations and, if so, by what mechanism, does not appear to be known.

The available evidence is conflicting. Halls and Young, speaking at a meeting of the Faculty of Radiologists, reported an instance in which the haustral pattern of folds seen during a barium enema examination of one patient's colon were recognizably the same in the same patient after an interval of 18 months. Pace and Williams (1969), in their study of the circular and longitudinal muscle layers of the colon, found no structural evidence to support the idea of fixed segmentation. However, they pointed out that fascicular linkages between the taeniae coli

Received for publication 24 March 1971.

${ }^{1}$ Address for correspondence: Nuffield Department of Clinical Medicine, the Radcliffe Infirmary, Oxford. and the circular muscle layer, especially those described by Lineback (1925), would tend to make the development of circular constrictions more likely at some sites than at others.

One way of deciding whether or not the segmental constrictions are permanent anatomical structures is to establish a fixed point on the colonic wall and to see if the constrictions always form at the same place in relation to it. The simplest marker to use for this purpose is the neck of a diverticulum as it avoids most of the objections that would apply to the use of metal clips on the gut wall. Observations should probably be confined to the transverse colon; that part of the bowel is seldom if ever involved in the sort of 'pre-diverticular' thickening of its wall so often found in the pelvic colon (Hughes, 1969), and diverticulosis there does not appear to upset the movement of contents.

This is an account of changes recorded in the fluorographic outline of colonic contents in the vicinity of diverticular markers. It relates, in particular, to three representative observations of subjects, whose bowel function at the time was normal, but similar changes were seen on a number of occasions in other subjects also.

\section{Materials and Method}

As part of an investigation of colonic propulsive movements (Ritchie, 1968), which has since been extended to include about 370 normal volunteers 
and patients attending the Radcliffe Infirmary, Oxford, each of the subjects was given $100 \mathrm{ml}$ of Micropaque barium sulphate suspension to drink at bedtime. After an interval of about 13 hours, the outlines of their colonic contents were observed over periods of between one and three hours by means of a $30-\mathrm{cm}$ image intensifier and time-lapse cinefluorography at 1 frame/minute.

\section{Results}

Diverticula were seen to be present in $56(15 \%)$ of these subjects and, in a proportion of the studies, segmental masses of opaque bowel contents gradually changed their position in relation to fixed points on the bowel wall which the diverticula marked. Many of the changes that were seen appeared to be due to the formation of fresh segmental constrictions and the elimination of those already present; some of them represented a progressive displacement of individual ring contractions in the long axis of the gut.

Figure 1 is made up of six exposures at twominute intervals from a time-lapse cinefluorographic film. They show changes occurring in the outline of a group of numbered segmental masses of opaque contents and in the constrictions separating them in the transverse colon of an otherwise normal subject. In the first frame a small diverticulum, indicated by the arrow, is seen lying in association with the proximal aspect of the mass of opaque bowel contents numbered 1 . Two minutes later there has been a circular contraction, chiefly affecting mass 1 , and the neck of the diverticulum has been closed off by thickening of the contracted muscle fibres surrounding it. At the same time, mass 2 has become merged with mass 3 through the elimination of the constriction between them. In the third frame

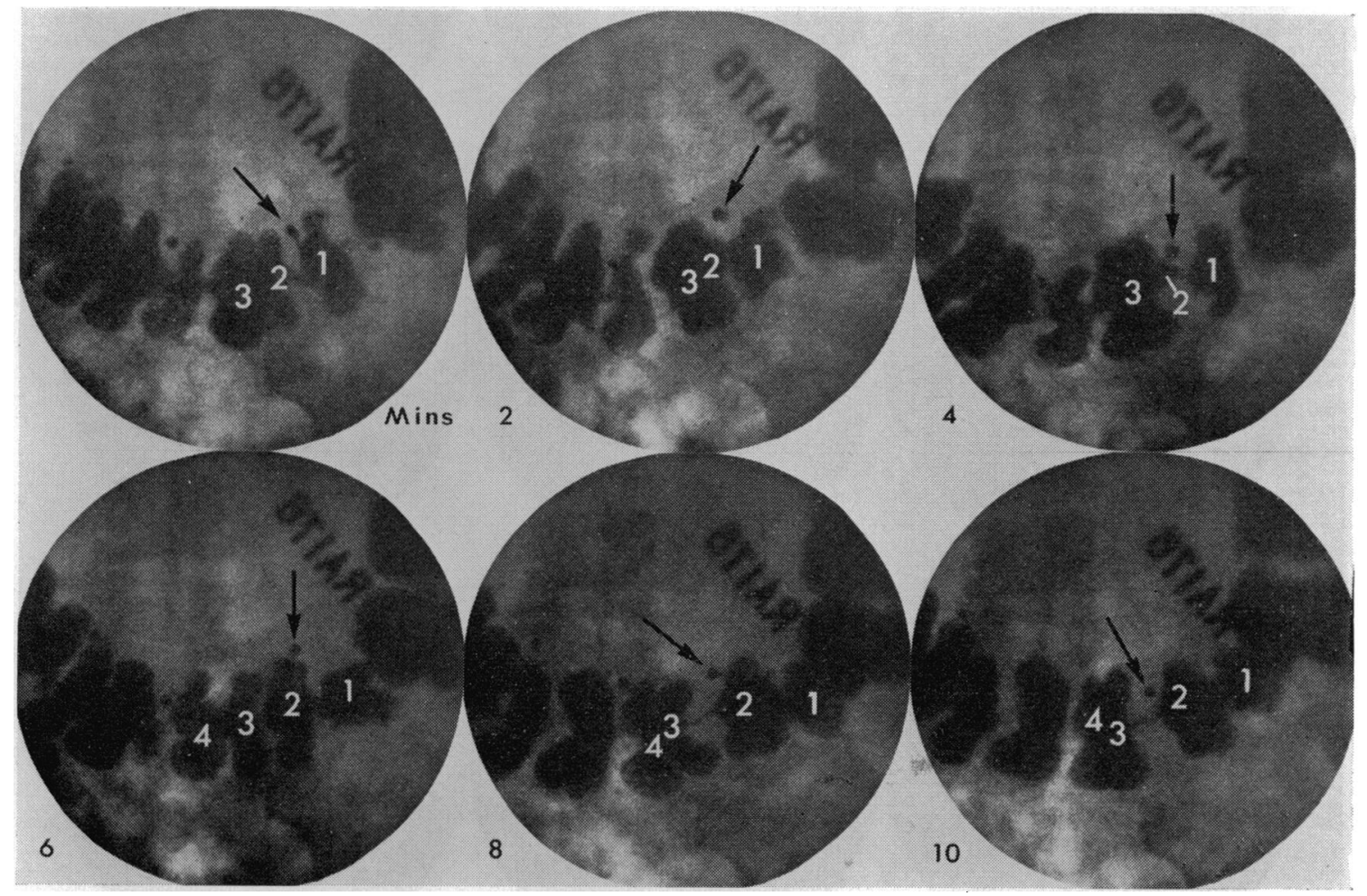

Fig. 1 Cinefluorograms at two-minute intervals showing changes in the position of segmental constrictions in relation to a diverticulum of the transverse colon.

In the first frame the contents of the diverticulum are in continuity with the segmental mass of opaque bowel contents numbered 1. Within two minutes the bowel wall surrounding mass 1 has contracted and the muscular activity has closed off the neck of the diverticulum. The annular constriction separating mass 2 from mass 3 has disappeared. When the constriction is re-formed four minutes later, it must have moved aborally because mass 2 is now lying opposite the diverticulum. In the final frame the opaque contents making up mass 2 have reached the section of bowel, just distal to the diverticulum, that was originally occupied by mass 1. 
the gut wall forming the proximal end of the constriction between mass 1 and mass 2 is beginning to relax; the diverticular neck can be seen passing through it and in process of re-opening. After another two minutes the segmental constriction between mass 2 and mass 3 has been restored. However, as mass 2 now lies opposite the diverticulum, the constriction must have been re-formed from a different group of circular muscle bands in the bowel wall. By the fifth frame the constriction has extended in both directions; mass 3 appears to be displaced proximally by the process and combines with mass 4 , whose characteristic sigmoid shape and separate diverticular marker have so far remained unchanged. Mass 2 moves distally; in the final frame it has reached the section of bowel that originally

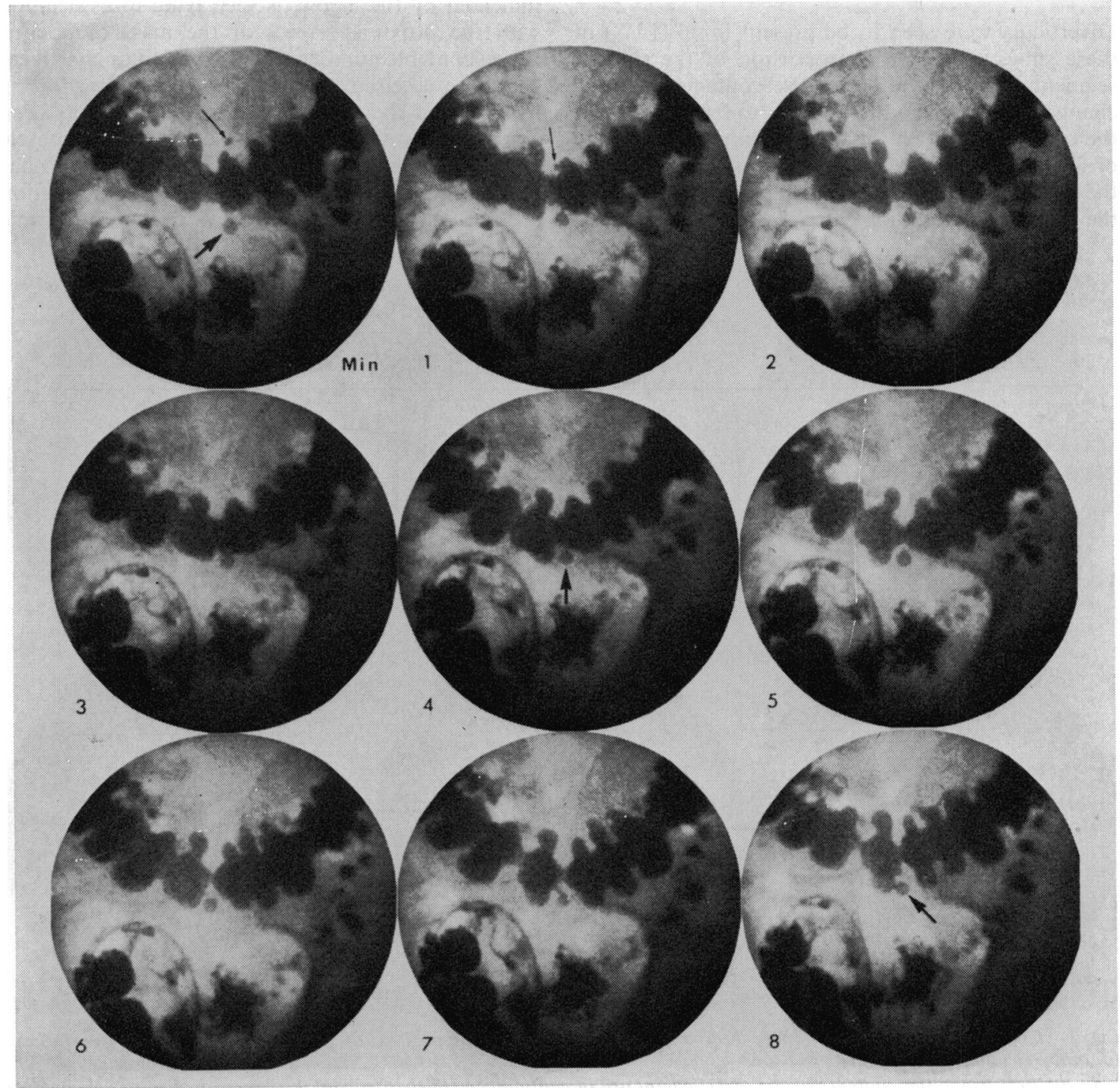

Fig. 2 Successive frames of time-lapse cine film at one-minute intervals showing the progressive aboral advance of a segmental constriction, and parts of the opaque masses of bowel contents on either side of it, past a diverticulum in the transverse colon, indicated by the larger arrow. A smaller diverticulum on the opposite side of the same segmental mass of opaque bowel contents (smaller arrow) also becomes involved in the advance of the constriction, and is obliterated by compression within the thickness of the contracting circular muscle. 


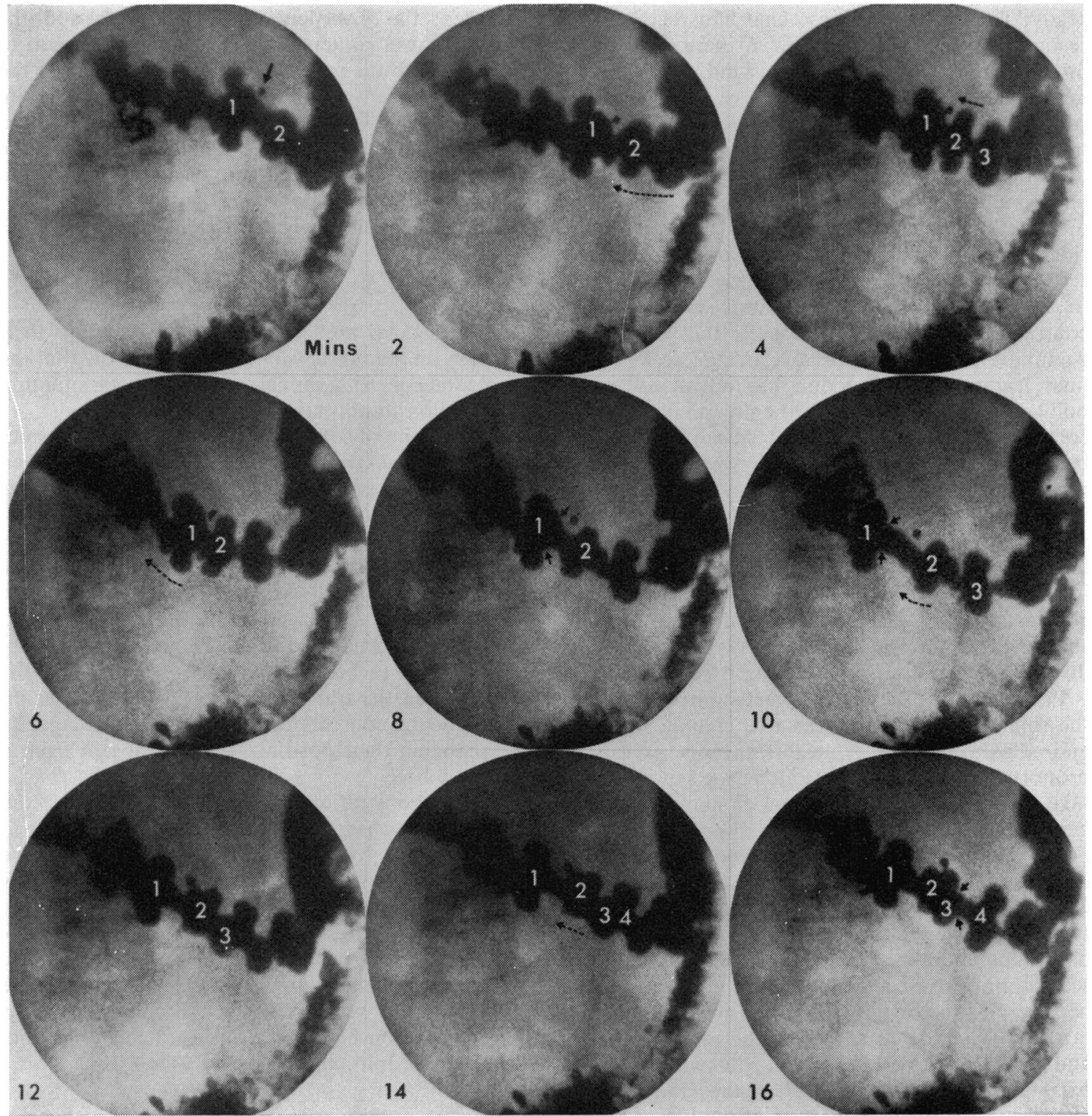

Fig. 3 Prints taken from a cinefluorographic film at two-minute intervals showing adoral progression of opaque contents in relation to a diverticulum of the transverse colon.

Over the first four minutes, the distal half of the transverse colon shortens. Then an annular constriction between the segmental masses labelled 1 and 2 begins to extend adorally, displacing mass 1 progressively towards the hepatic flexure. The neck of the diverticulum becomes involved and is occluded from the fourth frame; it is not freed again until, after another six minutes, the distal end of the constriction relaxes and this section of bowel is distended by the second segmental mass of opaque bowel contents. This is advancing in the same way past the diverticulum ahead of another retropulsive contraction wave when the series comes to an end. 
contained mass 1 and once again the neck of the diverticulum is becoming occluded by an encroaching circular contraction.

Figure 2, which is made up of prints from successive frames of time-lapse cine film relating to a second subject and occupies a period of eight minutes, illustrates a different kind of segmental movement. It shows a progressive change in the relationship of two diverticula containing opaque material-arrowed in the first frame-to the outline of two adjacent masses of opaque bowel contents and to the constriction between them. The larger diverticulum is at first positioned to the left-hand side of one segmental mass. Halfway through the series, four minutes later, the constriction is coming to lie opposite the diverticulum and increasing contraction of the circular muscle that forms it is beginning to close off the diverticular neck. In the final frame the constriction has advanced to the point where the contents of the diverticulum are in continuity with the right-hand side of the second barium mass; its neck, no longer involved in muscular contraction, is once again clear and open. The other, smaller, diverticulum marked by the smaller arrow in the first frame is similarly involved in the aboral progression of the contractile process; in this instance the increased thickness of the contracting ring of muscle occludes not only its shorter neck but also, within two minutes, the diverticular sac itself, obliterating it completely.

Progression of this kind, by extending one end of the ring contraction between two colonic segments and relaxing the other, will transport at least a proportion of their contents along the bowel. It can take place at varying rates between about $0 \cdot 1$ and 2 $\mathrm{cm} / \mathrm{min}$, and may travel in either direction. The nine prints making up Figure 3 are taken at two-minute intervals and show another small diverticular marker, arising from the transverse colon in a third subject. In this instance the two segmental masses of opaque bowel contents that are closest in relation to it are seen to move consecutively up the colon towards the hepatic flexure. In the first frame the diverticulum (arrowed) is situated alongside the mass labelled 1 and there is a broad constriction between this and mass 2. Over the next four minutes the zone of constriction is first folded, concertina-like, possibly by taenial contraction, and then shortened; the mass labelled 3 is also drawn proximally behind mass 2 . By the fourth frame, after six minutes, the constriction separating masses 1 and 2 has shortened till the pressure of their walls has closed off the neck of the diverticulum and is squeezing the diverticular sac itself out of shape between them. Then the zone of contracted circular muscle bands forming the constriction begins to extend adorally. During the next four minutes the circular contraction broadens progressively, carrying mass 1 ahead of it clear of the diverticulum. Between the tenth and twelfth minutes the muscular contraction proximal to mass 2 weakens; the diverticular neck re-opens and the opaque bowel contents making up mass 2 begin to move up into the relaxing section. After a pause the constriction between masses 1 and 2 shortens again. In the last two minutes mass 2 and what appears to be a part of mass 3 merge into a single unit; the constriction distal to it extends adorally as a second retropulsive contraction ring, pushing the barium contents ahead of it past the diverticulum.

\section{Discussion}

As diverticula are incapable of changing their position on the bowel wall, these films show that the segmental constrictions can move from one situation in the colon to another.

One cannot, of course, be certain that the dynamics of the colonic wall are completely normal when diverticula are present; in diverticular disease it has been shown (Morson, 1963) that the musculature of the distal bowel is frequently disordered. However, this is largely a theoretical objection. One of the principal effects of diverticular disease is to thicken the circular muscle layer of the pelvic colon into what appear to be fixed folds (Hughes, 1969, 1970). It is difficult to believe that such a condition, if it also affected the transverse colon, would confer on the segmental constrictions in that section a capacity for changing their position that was absent from a normal bowel.

The demonstration of movement confirms the preliminary observation previously reported in abstract form by Ritchie, Ardran, and Truelove (1969), which indicated that segmental constrictions of this kind might not be fixed anatomical structures; their progression along the bowel probably represents the basic mechanism of the peristaltic ripples, which were postulated by Alvarez (1967). Progressive movement of a series of segmental constrictions up the pelvic colon has been demonstrated by Ritchie, Truelove, Ardran, and Tuckey (1971).

This work was supported in part by a grant from the Medical Research Council and partly by the Nuffield Committee. I should also like to thank Drs Sidney Truelove and Gordon Ardran for their help.

\section{References}

Alvarez, W. C. (1967). Reverse peristalsis in bowel and stomach. Mod. Med. G.B., 12, $735-738$.

Hughes, L. E. (1969). Postmortem survey of diverticular disease of the colon. Part II. The muscular abnormality in the sigmoid colon. Gut, 10, 344-351. 
Hughes, L. E. (1970). A study of abnormal muscular patterns in diverticular disease of the colon using the polysiloxane foam enema. Gut, 11, 111-117.

Lineback, P. E. (1925). Studies on the musculature of the human colon with special reference to the taeniae. Amer. J. Anat., 36, 357-367.

Morson, B. C. (1963). The muscle abnormality in diverticular disease of the sigmoid colon. Brit. J. Radiol., 36, 385-392.
Pace, J. L., and Williams, I. (1969). Organisation of the muscular wall of the human colon. Gut, 10, 352-359.

Ritchie, J. A. (1968). Colonic motor activity and bowel function. Gut, 9, 442-456 and 502-511.

Ritchie, J. A., Ardran, G. M., and Truelove, S. C. (1969). Haustral movement in the human colon. Gut, 10, 1048.

Ritchie, J. A., Truelove, S. C., Ardran, G. M., and Tuckey, M. S. (1971). Propulsion and retropulsion of normal coloniz contents. Amer. J. dig. Dis. in press. 